\title{
Transverse Myelitis Secondary to SARS-CoV-2 Infection in a Pediatric Patient
}

\author{
Luis Del Carpio-Orantes ${ }^{1 *}$, Bernardo Ramírez-Orantes ${ }^{2}$, Carlos Humberto Aguilar-Arguello², \\ Gonzalo Antonio Neme Díaz and Pablo Valladares-Sánchez ${ }^{3}$
}

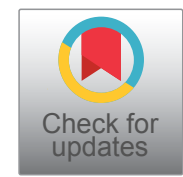

${ }^{1}$ Department of Internal Medicine, General Hospital 71, Veracruz Norte Delegation, Mexican Institute of Social Security, Mexico

${ }^{2}$ Department of Pediatric Infectology, Regional Hospital of High Specialty for Children "Dr. Rodolfo Nieto Padrón", Mexico ${ }^{3}$ Department of Pediatric Neurology, Regional Hospital of High Specialty for Children "Dr. Rodolfo Nieto Padrón", Mexico

\begin{abstract}
The clinical case of an 11-year-old male patients presented, treated at the Internal Medicine Service of the Hospital Regional de Alta Especialidad del Niño Dr. Rodolfo Nieto Padrón, Tabasco for presenting weakness of the upper and lower extremities of 4 days of evolution at the time of admission, with the history that 17 days prior to the onset of weakness, he had fever and dysgeusia treated only with antipyretic. Complementary studies were carried out such as a lumbar puncture which was normal, as well as a Magnetic resonance of the spine with which the diagnosis of transverse myelitis is reached and as an etiological agent SARS-CoV-2 since it presented positive IgG antibodies and negative IgM these observed In blood serum, no treatment directed at SARS-CoV-2 was given, only treatment with pulses of steroid for myelitis, after which the Patient evolved satisfactorily.
\end{abstract}

\section{Keywords}

Transverse Myelitis, SARS-CoV-2

\section{Introduction}

Transverse myelitis is a disorder characterized by the sudden development of a motor and sensory deficit. It can affect men and women of all races equally, but it has two periods of greatest incidence that are between 10 and 19-years and a second peak between 30 and 39-years [1]. Young children, ages 3 and under, develop spinal cord dysfunction that can last from several hours to a few days. They report a history of an infectious disease, generally of viral origin, or of an immunization, in the weeks prior to the development of their neurological difficulties. Loss of clinical function is often severe and can be complete. In these cases there is usually a slow recovery, but it is likely to be incomplete. In older children, the syndrome is a bit different. Although the onset is rapid, with loss of neurological function between 2 days and 2 weeks, recovery is faster and is more likely to be complete [2]. The picture presents as localized lumbar pain, paresthesia of sudden onset in the lower limbs with loss of sensation and paraparesis that can evolve to paraplegia, which is commonly added to bladder and bowel dysfunction. Coordination and sensitivity of the upper limbs can also be compromised $[3,4]$.
On many occasions it is difficult to establish the diagnosis since there are different ethylogy, among these are infectious (polio, Herpes zoster, HIV, Epstein barr, etc.), immunizations, immunologicals such as SLE, collagen, vascular or tumor diseases.

The diagnosis is based on an adequate interrogation and physical examination, which leads to suspicion to request Complementary studies such as MRI with gadolinium, lumbar puncture for analysis of cerebrospinal fluid, integrating the diagnostic criteria of TM.

Treatment is aimed at resolving the inflammatory

*Corresponding author: Luis Del Carpio-Orantes, Department of Internal Medicine, General Hospital 71, Veracruz Norte Delegation, Mexican Institute of Social Security, Veracruz, Mexico

Accepted: December 28, 2021

Published online: December 30, 2021

Citation: Carpio-Orantes LD, Ramírez-Orantes B, AguilarArguello $\mathrm{CH}$, et al. (2021) Transverse Myelitis Secondary to SARS-CoV-2 Infection in a Pediatric Patient. J Pediatr Neurol Neurosci 5(2):194-196 
process at the spinalcord level and detecting its progress. Treatment includes methylprednisolone, cyclophosphamide, or plasmapheresis [4].

\section{Presentation of the Clinical Case}

This is an 11-year-old male Patient who has a history that 3 weeks prior to admission presented fever and dysgeusia which was treated with antipyretic, without any other management, 4 days prior to admission presents gradual weakness of the lower extremities, with limited walking, presence of urinary and fecal incontinence, sensoryloss at the T5 level.

Physical examination revealed uncompromised cardiopulmonary, soft abdomen with peristalsis present without evidence of peritoneal irritation. On neurological examination, cranial nerves preserved, sensitivity with presence of the same up to T5 level, upper limb muscle strength $2 / 5$ on the Daniells scale, decreased palmar grasp, with $3 / 5$ distal muscle hypotrophy, preserved proximal muscle strength $5 / 5$, lower extremities with hyperreflexia, muscle strength $2 / 5$ on the Daniells scale, positive bilateral babinski, right patellar reflex abolished, left patellar reflex present but diminished, bilateral 3/5 dorsiflexion, no clonus, bilateral cremasteric reflex present but diminished. Hematic biometry, serum electrolytes, coagulation times, renal function, urinalysis, and erythrocyte sedimentation rate were performed, all within normal ranges.

It was decided to take a puncture for a CSF simple which was reported color less, 56 proteins, 59 glucose, rock water, 0 cells, negative culture for bacteria. A Magnetic resonance imaging of the spine with gadolinium was taken in which the presence of hyperintensity was observed in $\mathrm{T} 2$ sequences at the level of the anterior and posterior horns from C7 to T5 with a predominance of the anterior without increasing the medullary caliber. The spinal canal with transverse diameters and AP preserved radiological data of a transverse myelitis. There are no areas of bleeding, no masses, no extrinsic compression or alteration in morphology (Figure 1 and Figure 2).

Given a history of fever and dysgeusia, it was decided to take antibodies for SARS-CoV-2 in which positive IgG and negative IgM were obtained.

Pulse therapy is started with methylprednisolone boluses and subsequently maintenance doses at $1 \mathrm{mg} / \mathrm{kg} / \mathrm{day}$, recovery of sensation of the extremities is observed as well as progressive recovery of muscle strength, complete control of the anal and bladder sphincter was achieved, Physical rehabilitation therapy is added to the treatment provided by the kinesiology and physiatry service.

\section{Discussion}

Transverse myelitis is a disorder of multiple etiology and in many cases the cause is not identified.

In this time we have had to face a highly contagious Infection called SARS-CoV-2 which has become a pandemic, and of which we are still in the learning process discovering its pathophysiology and its effect on the different organs and

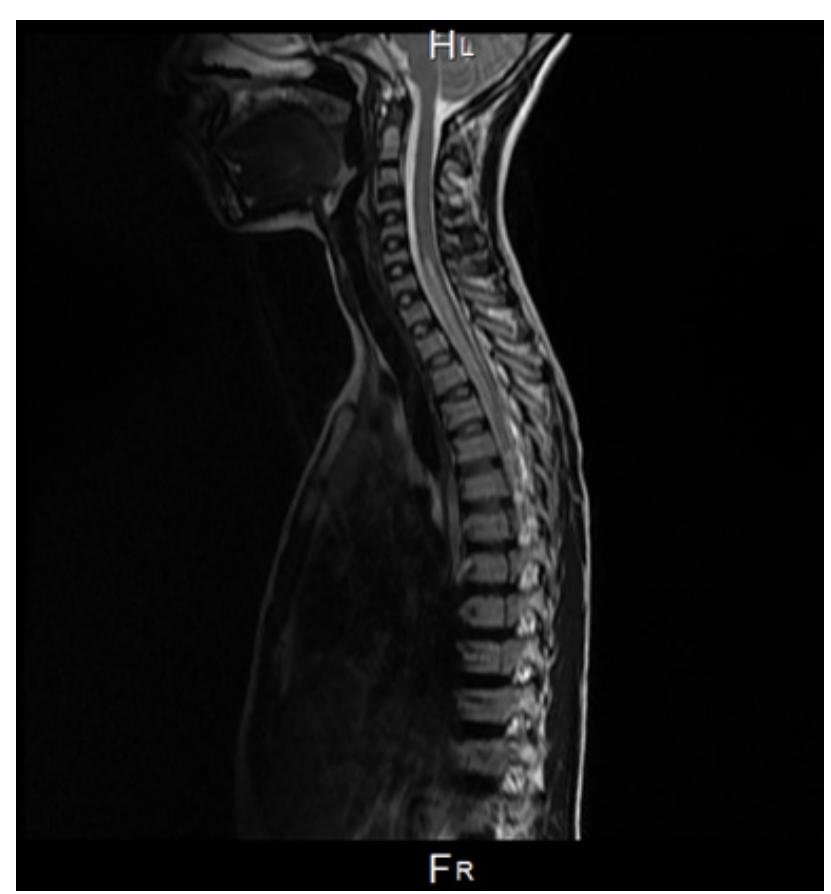

Figure 1: MRI with hyperintensity was observed in $\mathrm{T} 2$ sequences at the level of the anterior and posterior horns from C7 to T5.

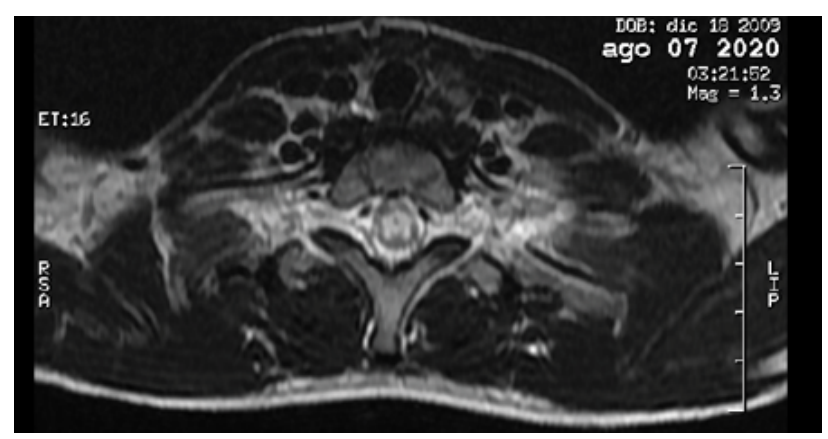

Figure 2: The spinal canal with transverse diameters and AP preserved radiological data of a transverse myelitis.

systems. We have also noticed that there may be sequelae weeks after the infectious process. The effects caused by the virus on the lower respiratory system are well known. It has also been seen that alterations can develop at the level of the central nervous system, these descriptions have been made of adult patients, but Little information is available in the pediatric environment.

In this precise case, it was posible to identify the etiological agent, the correct questioning and the adequate physical examination were key to orient the type of complementary study for the correct diagnosis and treatment as well as identification of the causative agent. MRI is essential for diagnosis but also to identify compressive lesions that simulate TM.

In our case, the MRI showed characteristic TM findings. And a relationship was observed with the infectious history that presented only fever and dysgeusia, which was suspected in SARS-CoV-2 and with positive IgG and negative IgM in 
hematic serum for SARS-CoV-2. With which the etiological agent was found.

As a treatment, steroids are the therapy of choice, and are aimed at reducing the inflammatory process at the spinal level and stopping the progression of the disease. Approximately 50 to $70 \%$ of patients have a partial or complete recovery. Treatment should include physical rehabilitation therapy to regain some lost functions.

Treatment is based on the steroid methylprednisolone at a dose of $30 \mathrm{mg} / \mathrm{kg} /$ day for 3 to 5 days. Our Patient was given IV pulsed steroid for 5 days, followed by oral steroid with prednisone for 4 weeks at a reduction dose with a favorable outcome. He continues with rehabilitation to improve his residual limitation.

\section{References}

1. Berman M, Feldman S, Alter M, et al. (1981) Acute transverse myelitis: incidence and etiologic considerations. Neurology 31: 966-971.

2. Robert $M$, Kliegman MD, Bonita F, et al. (2011) Nelson Tratado de Pediatría. (19 ${ }^{\text {th }}$ edn), 2182.

3. De Frohman EM, Wingerchuk DM (2010) Transverse mielitis. N Engl J Med 363: 564-572.

4. Cecilia Martínez, Hopsital Susana Lopez de Valencia, Popayan, et al. (2018) Mielitis transversa Análisis clínico. revisión de caso.

DOI: $10.36959 / 595 / 437$

Copyright: (c) 2021 Carpio-Orantes LD, et al. This is an open-access article distributed under the terms of the Creative Commons Attribution License, which permits unrestricted use, distribution, and reproduction in any medium, provided the original author and source are credited. 